\title{
ARTICLE \\ Somatic PIK3RI variation as a cause of vascular malformations and overgrowth
}

\author{
Catherine E. Cottrell ${ }^{1,2}$, Nicole R. Bender ${ }^{3}$, Michael T. Zimmermann ${ }^{4,5,6}$, Jonathan W. Heusel ${ }^{7,8}$, Meagan Corliss ${ }^{7}$, Michael J. Evenson ${ }^{7}$, \\ Vincent Magrini ${ }^{1}$, Donald J. Corsmeier ${ }^{1}$, Matthew Avenarius ${ }^{9}$, Jeffrey N. Dudley ${ }^{10,21}$, Jennifer J. Johnston ${ }^{10}$, Marjorie J. Lindhurst ${ }^{10}$, \\ Katinka Vigh-Conrad ${ }^{11}$, Olivia M. T. Davies ${ }^{12}$, Carrie C. Coughlin ${ }^{13}$, Ilona J. Frieden ${ }^{14}$, Megha Tollefson ${ }^{15}$, Andrea L. Zaenglein ${ }^{16}$, \\ Heather Ciliberto ${ }^{17}$, Laura L. Tosi ${ }^{18}$, Robert K. Semple ${ }^{19}$, Leslie G. Biesecker ${ }^{10}$ and Beth A. Drolet (iD ${ }^{20 凶}$
}

PURPOSE: Somatic activating variants in the PI3K-AKT pathway cause vascular malformations with and without overgrowth. We previously reported an individual with capillary and lymphatic malformation harboring a pathogenic somatic variant in PIK3R1, which encodes three PI3K complex regulatory subunits. Here, we investigate PIK3R1 in a large cohort with vascular anomalies and identify an additional 16 individuals with somatic mosaic variants in PIK3R1.

METHODS: Affected tissue from individuals with vascular lesions and overgrowth recruited from a multisite collaborative network was studied. Next-generation sequencing targeting coding regions of cell-signaling and cancer-associated genes was performed followed by assessment of variant pathogenicity.

RESULTS: The phenotypic and variant spectrum associated with somatic variation in PIK3R1 is reported herein. Variants occurred in the inter-SH2 or N-terminal SH2 domains of all three PIK3R1 protein products. Phenotypic features overlapped those of the PIK3CArelated overgrowth spectrum (PROS). These overlapping features included mixed vascular malformations, sandal toe gap deformity with macrodactyly, lymphatic malformations, venous ectasias, and overgrowth of soft tissue or bone.

CONCLUSION: Somatic PIK3R1 variants sharing attributes with cancer-associated variants cause complex vascular malformations and overgrowth. The PIK3R1-associated phenotypic spectrum overlaps with PROS. These data extend understanding of the diverse phenotypic spectrum attributable to genetic variation in the PI3K-AKT pathway.

Genetics in Medicine (2021) 23:1882-1888; https://doi.org/10.1038/s41436-021-01211-z

\section{INTRODUCTION}

Vascular malformations and the overgrowth syndromes of which they are commonly a part constitute a heterogeneous group of congenital malformations that lead to significant morbidity and disfigurement. Next-generation sequencing (NGS) has become an important tool in genomic investigation of these syndromes, allowing improved molecular characterization and diagnosis. Developments in NGS technology have enabled successful discovery of disease-associated somatic variation within affected tissue. ${ }^{1,2}$ It is now recognized that vascular malformations and overgrowth demonstrate some shared genetic variation with cancer, with the phosphoinositide 3-kinase (PI3K)-AKT growth signaling pathway commonly dysregulated in both sets of disease. ${ }^{3-5}$

Class I phosphatidylinositol 3-kinases (PI3K) function as heterodimers composed of a catalytic and a regulatory subunit and serve as intracellular signal transducers that convert phosphoinositide $(4,5)$-bisphosphate into phosphoinositide $(3,4,5)$-trisphosphate (PIP3).
PIP3 generation triggers activation of downstream effectors including PDK1 and then AKT, which promote cell growth and survival. ${ }^{6}$ Somatic mosaic activating variants in PIK3CA, encoding the $\mathrm{p} 110 \mathrm{a}$ catalytic subunit of the PI3K heterodimer, have been well described in vascular malformations and overgrowth syndromes. ${ }^{7}$ Among the regulatory subunits of $\mathrm{PI} 3 \mathrm{~K}, \mathrm{PIK} 3 \mathrm{R} 1$ encodes three distinct protein products (p85a, p55a and p50a), generated through alternative splicing. These products form obligate heterodimers with PIK3CA, stabilizing and inhibiting it in the basal state, while mediating its binding to activated receptor tyrosine kinases and its subsequent activation. ${ }^{8}$ PIK3R1 also negatively regulates the PI3K pathway by stabilizing the phosphatase PTEN, itself a tumor suppressor. ${ }^{9}$

PIK3R1 variants that fail to inhibit $\mathrm{p} 110 \mathrm{a}$ activity, usually by disruption of the inter-SH2 domain, cause constitutive PI3K pathway activation, and are enriched in cancers, albeit much less commonly than PIK3CA variants. Given the numerous variants identified in PI3K pathway components (PIK3CA, AKT1,

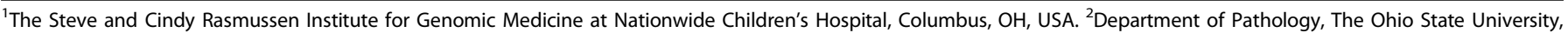

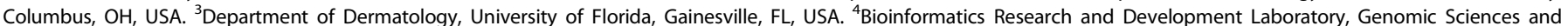

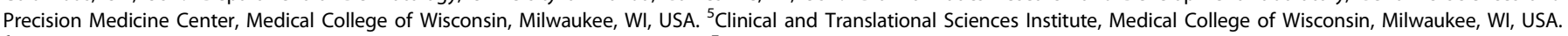

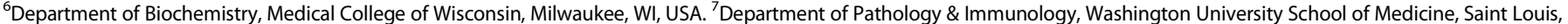

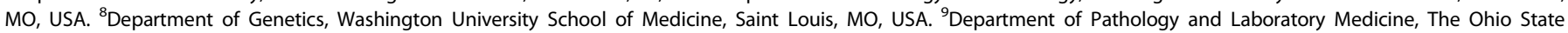

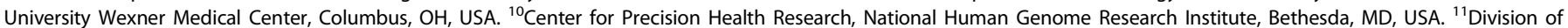

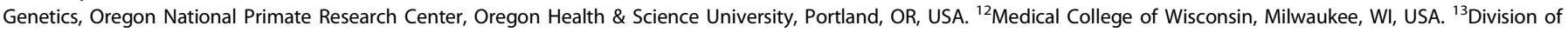

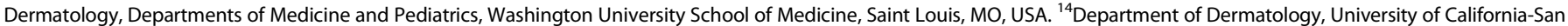

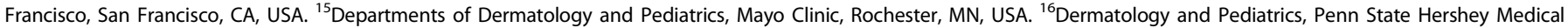

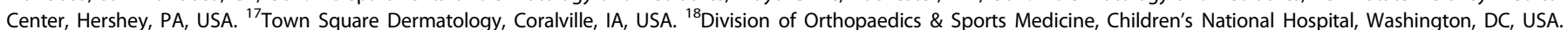

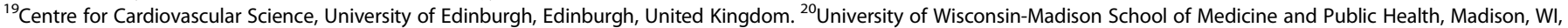

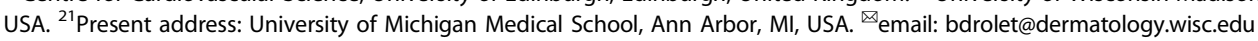


and PTEN) in overgrowth, PIK3R1 is an excellent candidate gene for vascular malformations and overgrowth. We used targeted, high-depth NGS to analyze Affected tissue from a cohort of individuals with vascular malformations, and thereby extend understanding of the role played by PIK3R1 in these clinically important disorders.

\section{MATERIALS AND METHODS}

Study cohort

The study was authorized by the institutional review board of participating institutions. Individuals described herein were identified to harbor a variant in PIK3R1 amid the setting of apparently mosaic disease and were derived from one of three cohorts that together enabled the assemblage of variant and phenotype data within this described study cohort. The primary cohort is a multisite network coordinated through the Pediatric Dermatology Research Alliance (PeDRA) $)^{3}$ enrolling patients of any age with vascular anomalies and overgrowth. Specimens were available on 108 patients consisting of 3-4 mm skin punch biopsy samples from affected tissue or a paraffin-embedded sample of affected tissue from previous excisions. Within the PeDRA cohort, ten PIK3R1 variant-positive individuals were identified. Among a National Institutes of Health (NIH) cohort of 297 individuals ascertained for heterogeneous manifestations of vascular anomalies and overgrowth, two PIK3R1 variant-positive individuals were identified. The $\mathrm{NIH}$ cohort eligibility criteria were apparently mosaic (segmental) overgrowth of extra-central nervous system (CNS) organs/ tissue. Individuals may or may not have had CNS manifestations, but those with CNS manifestations alone were not included, nor were those with vascular anomalies alone without other manifestations of overgrowth. Samples collected for sequencing included either punch skin biopsies of apparently affected tissues or excisional biopsies collected at the time of surgery. Of necessity, these samples were highly heterogeneous in nature and number, based on the clinical needs and limitations of the individuals. The Genomics and Pathology Services (GPS) at Washington University School of Medicine cohort consisted of five PIK3R1 variant-positive individuals ascertained from a total of 343 individuals assayed clinically for suspected disorders of somatic mosaicism, including but not limited to overgrowth and vascular malformation. Where available, clinical data were retrospectively collected and included medical history, dermatologic and musculoskeletal exams, and clinical and radiologic images. Available clinical and radiologic images were reviewed centrally. The NIH study was reviewed and approved by the National Human Genome Research Institute (NHGRI) institutional review board (IRB), protocol number 94-HG-0132.

\section{Sequencing methodologies}

NGS was utilized in all centers to identify genetic variation (Supplemental methods). DNA was extracted from affected fresh frozen tissue (FT), cultured tissue (CT), or from formalin-fixed paraffin-embedded (FFPE) blocks of previously excised affected tissue.

\section{RESULTS}

Identification of pathogenic variants in PIK3R1

PIK3R1 variants were detected in tissue of 17 individuals within the study cohort, and were observed at a reduced variant allele frequency/fraction (VAF) consistent with a somatic etiology, with most detected at less than $10 \%$ (Table 1). The identified variants included missense and insertion-deletion (indel) variants within the SH2 $(n=1)$ and PI3K_P85_iSH2 $(n=16)$ domains of PIK3R1 and overlapped regions harboring known hotspots seen in cancers (Fig. 1). ${ }^{10-12}$ Notably, the variants detected in vascular malformation and overgrowth occur in domains that are common to all PIK3R1 products (p85a, p55a, and p50a). Recurrent variation was detected among this cohort resulting in nine unique variants at the level of the coding sequence, and seven unique variants at the level of the predicted protein consequence. Indel events comprised three in-frame deletions and four splice-site alterations.
Variant attributes

Variants were interpreted using a modification of the American College of Medical Genetics and Genomics and the Association for Molecular Pathology (ACMG/AMP) standards and guidelines for variant interpretation as described in the Supplemental methods. ${ }^{13}$ Variants were considered to be of somatic origin if the variant allele was observed at a diminished VAF or a variant was present at differing frequencies among tissues from the same individual. Variant attributes considered when assessing for pathogenicity included variant location within the gene or protein product (hotspot or domain), occurrence and frequency within this cohort, and within the wider setting of human disease, as well as variant type and predicted impact on the protein product. Among the detected variants, 15 were classified as pathogenic ( 5 of these being unique at the level of the predicted amino acid change), with 2 classified as likely pathogenic.

\section{Clinical phenotype in individuals with PIK3R1 variants}

Expert-reviewed clinical images and data were available for 12 individuals harboring PIK3R1 variants, and their phenotypes were similar to those attributed to somatic mosaic hotspot variants in PIK3CA. Most patients had red vascular stains (10/12), venous ectasias or engorgement (11/12), and mild soft tissue or bone overgrowth (11/12). Four individuals had sandal toe gap deformities of the foot with mild macrodactyly of the second toe (Table 2, Fig. 2). Other clinical features noted included developmental delay, cutaneous syndactyly, and lipoma/fatty tissue overgrowth. Among individuals in whom extensive phenotype information was available (PeDRA cohort, $n=10$; NIH cohort, $n=2$ ), seizures, macrocephaly, or hydrocephalus were not described. Individuals had been previously diagnosed with various acronyms or eponyms including Klippel-Trenaunay syndrome, CLOVES syndrome, and PROS.

\section{DISCUSSION}

PIK3R1 is ubiquitously expressed and has important roles in physiology and disease. It encodes the p85a, p55a, and p50a regulatory subunits of class $1 \mathrm{~A} \mathrm{PI3K}$, which bind tightly to any of the $\mathrm{p} 110 \mathrm{a}, \beta$, or $\delta$ catalytic subunits. It is the $\mathrm{p} 110 \mathrm{a}$ subunit, encoded by PIK3CA, that is by far the most commonly mutated and activated in cancer and overgrowth syndromes. PI3K transduces cell surface activation of receptor tyrosine kinase growth factor and hormone receptors into downstream activation of AKT and other pathways to regulate cell metabolism, size, differentiation, proliferation, migration, and apoptosis. ${ }^{14,15}$ The $\mathrm{PI} 3 \mathrm{~K} / \mathrm{AKT}$ pathway is constitutively activated in affected tissue from many vascular malformations, ${ }^{1,16,17}$ primarily through mosaic variation in $P I K 3 C A$, as in cancer.

PIK3R1 variation in the setting of vascular malformation with overgrowth has been rarely reported. We previously described a single individual harboring a somatic mosaic PIK3R1 variant, p.(Lys567Glu), with capillary and lymphatic malformation, and leg length discrepancy (individual 4 as referenced in this cohort). ${ }^{3}$ A further patient harboring a PIK3R1 iSH2 domain variant, p.(Asn564Lys), was reported to have macrocephaly, tracheomalacia, and cardiovascular malformation described by the authors as in the context of megalencephaly-capillary malformation (MCAP) syndrome, as well as recurrent infections in keeping with activated PI3Kdelta syndrome 2 (APDS2). ${ }^{18}$ We confirm in a large cohort that somatic mosaic PIK3R1 variants are a significant cause of vascular malformation and overgrowth. The phenotypes observed in individuals with somatic mosaic variants in PIK3R1 are similar to those associated with mosaic PIK3CA hotspot variants. All individuals with mosaic PIK3R1 variants had red-purple, geographic vascular stains, most with associated venous engorgement, venous prominence, and soft tissue or bone overgrowth, but this tended to be mild and relatively uniform. One individual with a unique variant 
C.E. Cottrell et al.

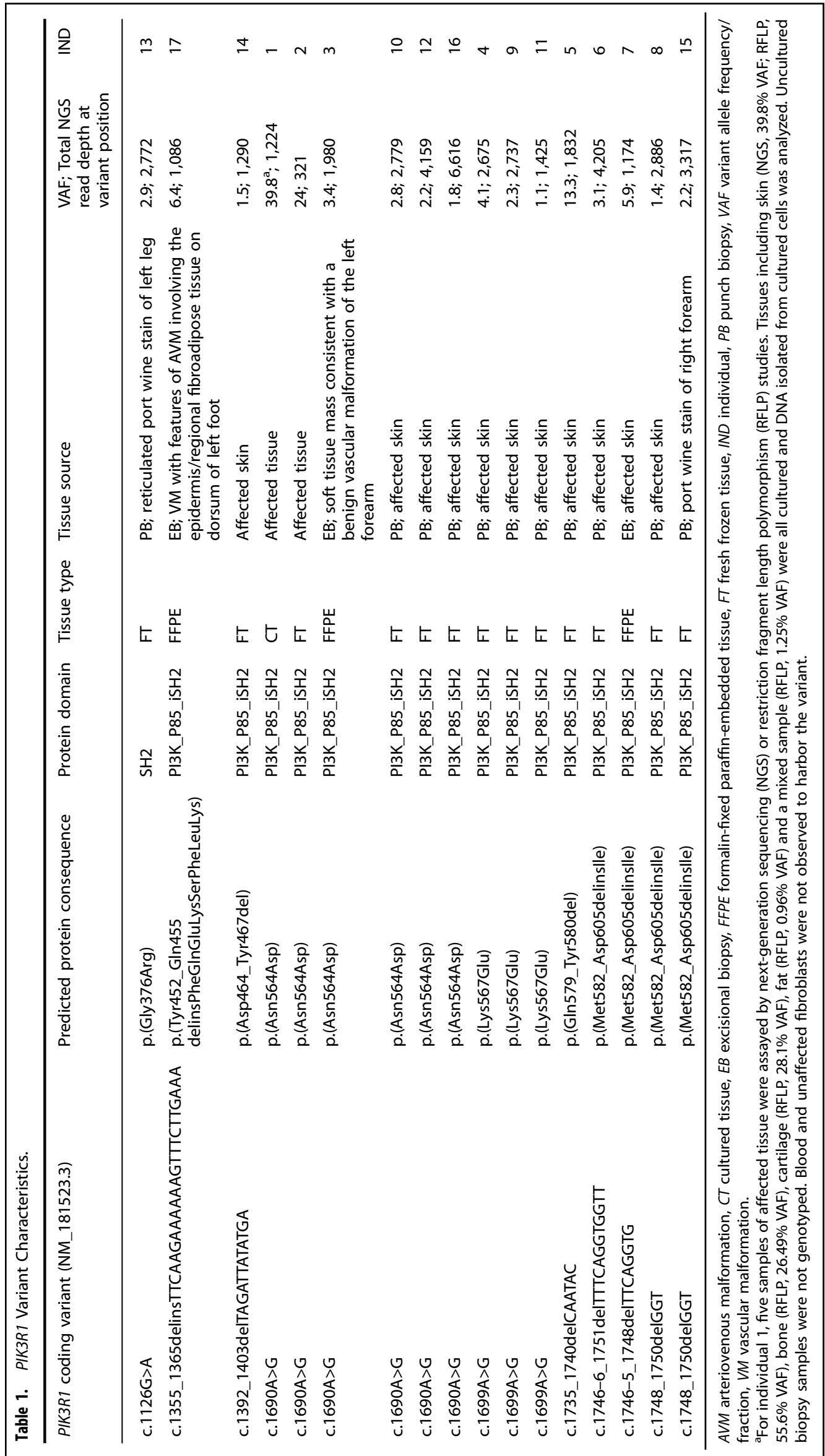




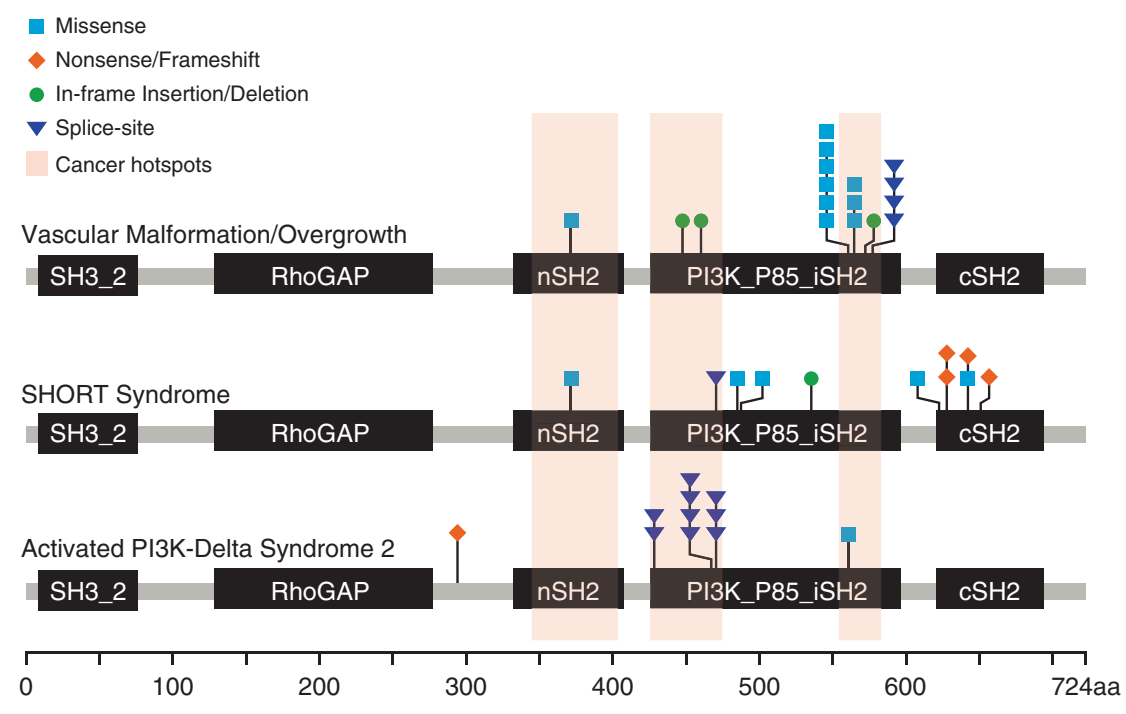

PIK3R1 NP_852664.1

Fig. 1 Domain structure of the longest PIK3R1 protein product showing distribution of monogenic disease-associated variants. Variants described in this study in association with a vascular malformation/overgrowth phenotype (top row). Variants described as pathogenic or likely pathogenic in the ClinVar database (accessed 13 November 2020 and filtered to encompass only variation less than 51 bp and with a described genetic condition) associated with SHORT syndrome (middle row) or activated PI3K-delta syndrome 2 (bottom row).

within the N-terminal SH2 domain, p.(Gly376Arg), had a light pink reticulate vascular stain with associated limb undergrowth. Residue 376 has been designated as a cancer hotspot, with the variant itself, p.(Gly376Arg), functionally characterized as capable of inducing in vitro oncogenic transformation and activation of p110a. ${ }^{19,20}$ In total, the observed clinical features among the individuals assembled within our cohort suggest that somatic mosaic variants in PIK3R1 activate the PI3K pathway; however, the degree of activation, particularly in comparison to disease-associated variation in PIK3CA, requires further study.

As is the case for somatic mosaic overgrowth-associated variants in PIK3CA and AKT1, mosaic PIK3R1 variants are also found in cancer. ${ }^{21}$ PIK3CA is more commonly altered in cancer in comparison to PIK3R1. Among 181 studies with nonredundant samples curated in cBioPortal encompassing in total 47,580 samples, $10.3 \%$ harbored a PIK3CA variant, as opposed to $2.1 \%$ for PIK3R1 (date accessed 11 November 2020). Among this curated data set, in-frame variants were more common in PIK3R1 $(22.2 \%$ of all variants) than in PIK3CA (2.5\% of all variants). Similarly, in our PeDRA multisite network with vascular anomalies and overgrowth, PIK3R1 variants were less common $(9.2 \%, 10 / 108)$ than variants in PIK3CA (39.8\%, 43/108 individuals), with notable enrichment of in-frame variants in PIK3R1 (Supplemental Fig. 1).

Constitutional variants in PIK3R1 have been shown to exhibit striking genotype-phenotype correlation. Most pertinent to this study, variants disrupting canonical splicing of exon 11 and leading to in-frame deletions in the $\mathrm{N}$-terminal of the inter-SH2 domain cause APDS2, an immunodeficiency characterized by recurrent infections and lymphoproliferation. ${ }^{22}$ Hyperactivation of $\mathrm{PI} 3 \mathrm{~K}$ signaling in APDS2 has been demonstrated in lymphocytes, yet despite ubiquitous expression of the pathogenic variant, associated overgrowth has been exceedingly rarely described. ${ }^{18,23}$ In vitro studies have shown that APDS2 variants in PIK3R1 cause distinct patterns of hyperactivation of $\mathrm{p} 110 \delta$, the dominant lymphocyte catalytic subunit, and $\mathrm{p} 110 \mathrm{a}$, the ubiquitous growth-promoting subunit, based on subtle differences in the inhibitory molecular interactions of the regulatory and catalytic subunits: although both are hyperactivated, basal hyperactivation of $p 110 \delta$ was greater than 300 -fold, while basal activation of p110a was only twofold. ${ }^{24}$ This establishes that subtle differences in molecular interactions at the dynamic interface of regulatory and catalytic subunits can have major effects on the pattern of biochemical activation of mutant holoenzyme. This has yet to be studied for vascular and overgrowthrelated PIK3R1 variants, but it is plausible that these, too, result in a distinct profile of biochemical activation of different catalytic subunits. A full account of biochemical differences will also have to address any effects of variations in PI3K subunit expression and stoichiometry in various tissues, which is known to modulate PI3K activity.

In the aforementioned individual described with features of APDS2 and MCAP, the pathogenic heterozygous PIK3R1 variant, $\mathrm{p}$. (Asn564Lys), was associated with mildly increased lymphocyte AKT phosphorylation. ${ }^{18}$ A different missense change at codon 564, p.(Asn564Asp), was the most frequently detected variant in our vascular malformation and overgrowth cohort and is also described in cancer. Interestingly, in biochemical studies it has been shown to increase basal activity of $\mathrm{p} 110 \mathrm{a}$ and $\beta$ significantly more than $\delta$, a pattern opposite to that described for the APDS2 variant, albeit in a different experimental paradigm. ${ }^{25}$ Overt clinical manifestations of immune dysregulation were not identified in any of the individuals within our cohort, however; most patients did not undergo systematic laboratory evaluation for abnormalities in B cells and T cells.

In contrast to APDS2, constitutional PIK3R1 loss-of-function variants, predominantly in the C-terminal $\mathrm{SH} 2$ domain, have been shown to cause SHORT syndrome (short stature-hyperextensibilityhernia-ocular depression-Rieger anomaly-teething delay). ${ }^{26}$ These variants disrupt association of PI3K holoenzyme with activated RTKs, leading to downstream hypostimulation of the PI3K/AKT axis in response to ligand stimulation. The phenotype is correspondingly the "inverse" of vascular malformations and overgrowth, including intrauterine growth restriction, lipoatrophy, and insulin resistance/ diabetes. ${ }^{27}$ Of note, very rare reports of phenotypic overlap have been described between APDS2 and SHORT syndrome. ${ }^{28}$ In principle, these findings suggest that there are largely distinct spectra of PIK3R1 variants associated with these three disorders. Biochemical studies demonstrate that differences between SHORT syndrome and APDS2 are attributable to different profiles of activation or repression of PIK3CA and PIK3CD by mutant PIK3R1 products. 


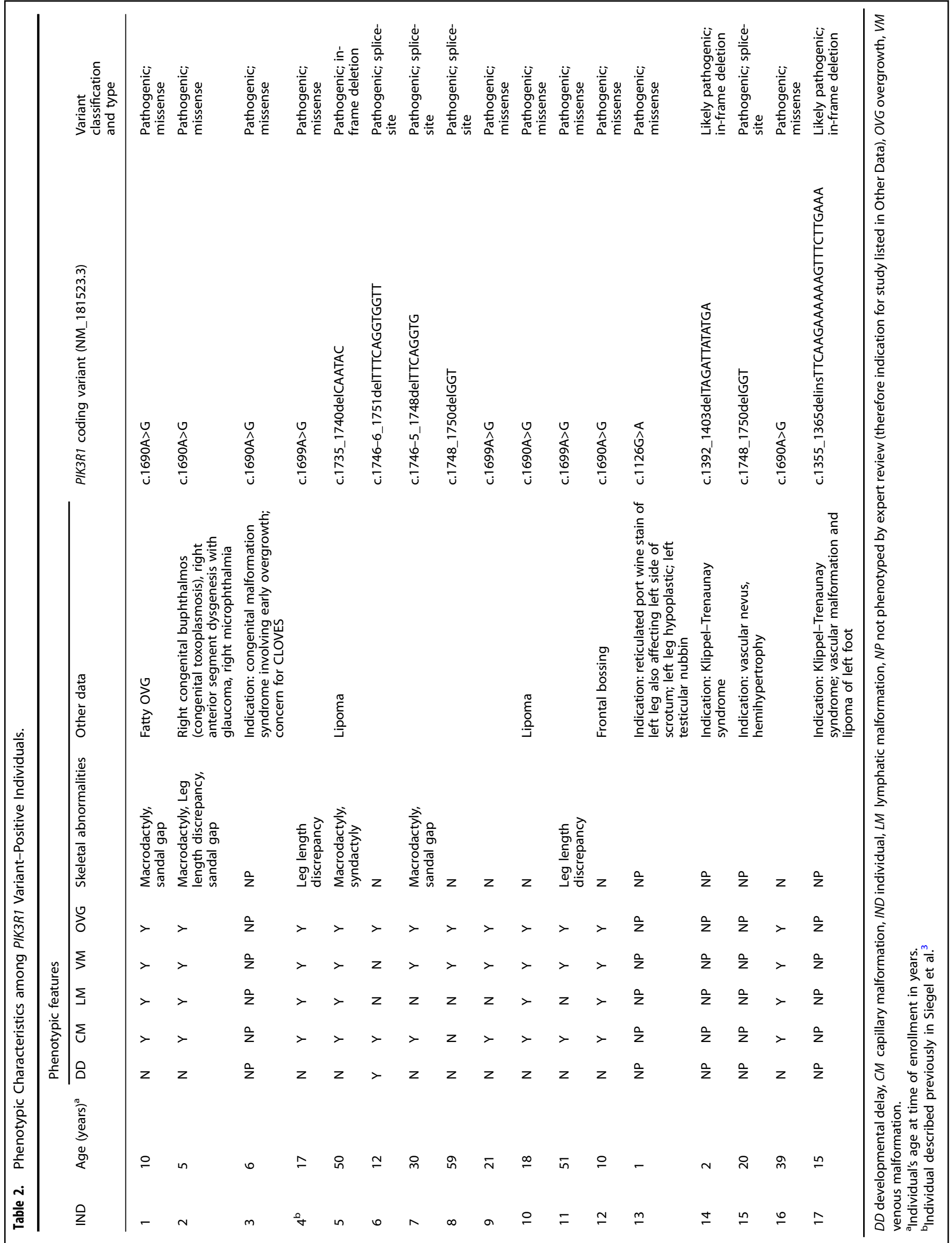




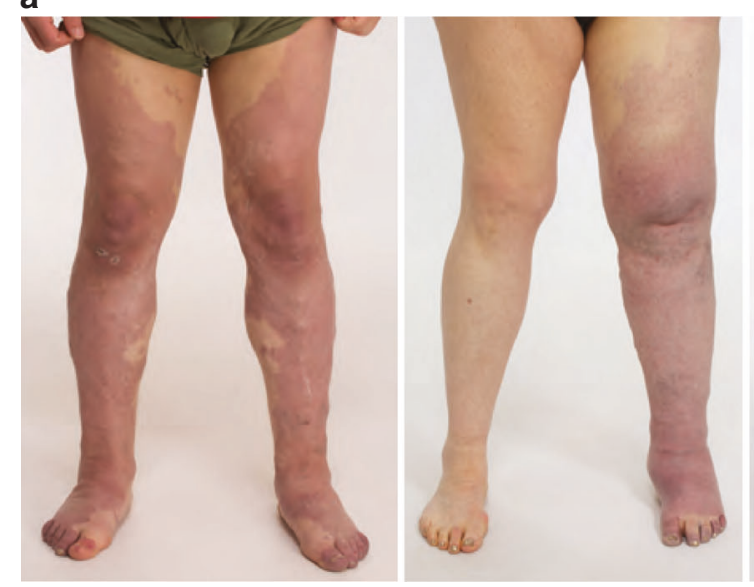

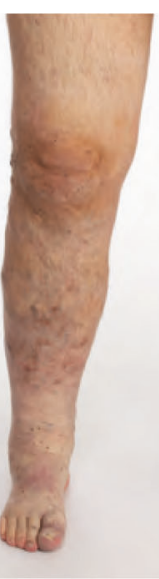

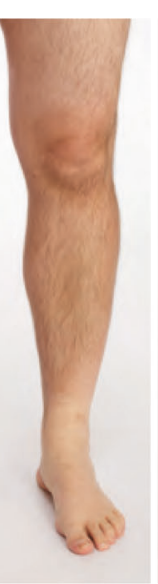

b

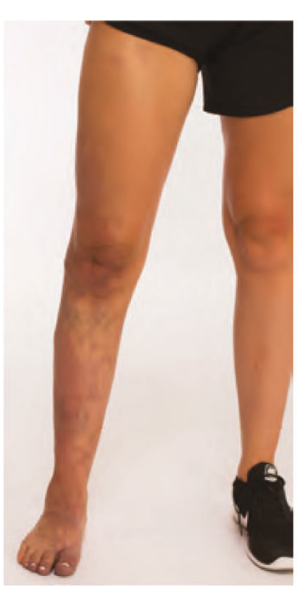

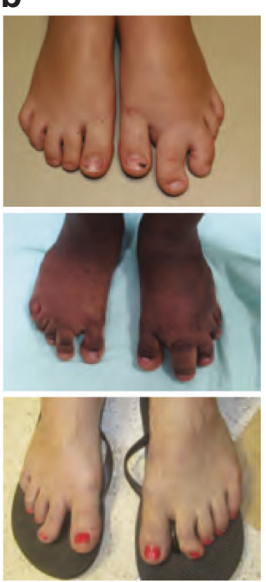

Fig. 2 Patient photographs of characteristic clinical phenotype. (a) Capillary venous malformation with limb overgrowth (Klippel-Trenaunay). (b) Macrodactyly with sandal toe deformity.

The mutational spectrum of PIK3R1-related somatic mosaic overgrowth is largely distinct from the constitutional PIK3R1-related disorders, and no apparent loss-of-function variants were seen among 17 unrelated affected individuals. Recurrent missense variants were identified, with one variant observed in affected tissue from three individuals, p.(Lys567Glu), and one variant in six individuals, p.(Asn564Asp). Indel variants were a frequent observation within this cohort with $7 / 17(41 \%)$ variants of this type detected. These included three predicted in-frame events and four splice-site alterations, the latter recurrently located at the intron 13/exon 14 junction (NM_181523.3) (Supplemental Fig. 2, Supplemental Table I). Due to the genomic architecture in this region, codon 582, which encodes a methionine, is split across exons 13 and 14. Furthermore, the intron 13/exon 14 junction consists of a spanning GGT sequence that is repeated once in exon 14. Genomic complexity and variability in published variant descriptions within this region further confound interpretation. Based solely on the observed sequencing data and established bioinformatic and nomenclature conventions, we would describe one such variant, c.1748_1750del, as predicted to encode p.(Trp583del). Notably, this variant has been reported previously in cancer literature and databases described as Trp583del, or as a splice variant affecting methionine codon $582 .^{10,29,30}$ In cancer studies, RNA sequencing demonstrates that variably sized indel splice variants impacting the splice acceptor (described as M582_splice) result in in-frame exon 14 skipping. ${ }^{11}$ Based on these data, the protein consequence of these exon 14 skipping variants should be described as p.(Met582_Asp605delinslle).

Both simple and complex indels are enriched in PIK3R1 at recurrent genomic regions defined as hotspots occurring within discrete clusters within the $\mathrm{SH} 2$ and inter-SH2 domains, with demonstrated statistical significance observed from large cancer data sets (Supplemental Table I) (cancerhotspots.org). ${ }^{1,12,31}$ One such variant in our cohort, p.(GIn579_Tyr580del), has been shown to demonstrate nearly twofold increased activity as measured by an in vitro phosphatidylinositol phosphorylation assay, retaining p110a binding but losing inhibitory activity. ${ }^{25,32}$ Further studies to establish the activation pattern conferred by PIK3R1 variants associated with vascular malformations and overgrowth are needed. Moreover, characterization of PIK3R1 variants and functional impact are of particular importance in consideration of treatment. The application of targeted therapeutics has been previously investigated in the setting of vascular malformation and somatic overgrowth. Importantly, studies of alpelisib, a targeted inhibitor of p110a, have demonstrated efficacy in PROS with attenuation of disease symptomatology and may reasonably be considered for further study in the setting of PIK3R1 variation. ${ }^{33}$

The somatic mosaic PIK3R1 variants we describe in vascular malformations and overgrowth further extend our understanding of PIK3R1, whose genetic perturbation produces pleiotropic manifestations. Several features of the vascular phenotype are the reverse of SHORT syndrome, displaying vascular and soft tissue overgrowth as opposed to short stature, reduced adipose tissue, and tissue underdevelopment observed in SHORT syndrome. Divergent clinical phenotypes are determined by the nature of the PIK3R1 alteration, the specificity of the consequences for PIK3Ca- and PIK3C $\delta$ mediated effects, and the timing and distribution of the alteration during embryogenesis. Disease-associated PIK3R1 variants are enriched for indel events in both cancer and vascular anomalies with overgrowth. Detection of such variants is bioinformatically challenging, and further complicated by nonstandardized annotation of the variant nomenclature. As such, these indel events are subject to ascertainment bias with additional study needed to discern frequency. Furthermore, studies to elucidate the molecular mechanisms underlying pathogenic variation of PIK3R1 with widely disparate clinical phenotypes, including both under- and overgrowth syndromes and immunoregulation, will unify our understanding of this critical cellular proliferation pathway and provide further insight into treatment.

\section{DATA AVAILABILITY}

Variant data have been deposited into the ClinVar database (https://www.ncbi.nlm. nih.gov/clinvar/) with the following submission identifiers: SCV001478395, SCV001478396, SCV001478397, SCV001478398, SCV001478399, SCV001478400, SCV001478401, SCV001478402, SCV001478403.

Received: 14 December 2020; Revised: 23 April 2021; Accepted: 4 May 2021;

Published online: 26 May 2021

\section{REFERENCES}

1. Lindhurst, M. J. et al. A mosaic activating mutation in AKT1 associated with the Proteus syndrome. N. Engl. J. Med. 365, 611-619 (2011).

2. Cottrell, C. E. et al. Validation of a next-generation sequencing assay for clinical molecular oncology. J. Mol. Diagn. 16, 89-105 (2014).

3. Siegel, D. H. et al. Analyzing the genetic spectrum of vascular anomalies with overgrowth via cancer genomics. J. Invest. Dermatol. 138, 957-967 (2018). 
4. Mirzaa, G. M., Riviere, J. B. \& Dobyns, W. B. Megalencephaly syndromes and activating mutations in the PI3K-AKT pathway: MPPH and MCAP. Am. J. Med. Genet. C Semin. Med. Genet. 163c, 122-130 (2013).

5. Keppler-Noreuil, K. M. et al. Clinical delineation and natural history of the PIK3CArelated overgrowth spectrum. Am. J. Med. Genet. A. 164a, 1713-1733 (2014).

6. Hopkins, B. D., Goncalves, M. D. \& Cantley, L. C. Insulin-PI3K signalling: an evolutionarily insulated metabolic driver of cancer. Nat. Rev. Endocrinol. 16, 276-283 (2020).

7. Madsen, R. R., Vanhaesebroeck, B. \& Semple, R. K. Cancer-associated PIK3CA mutations in overgrowth disorders. Trends Mol. Med. 24, 856-870 (2018).

8. Vadas, O., Burke, J. E., Zhang, X., Berndt, A. \& Williams, R. L. Structural basis for activation and inhibition of class I phosphoinositide 3-kinases. Sci. Signal. 4, re2 (2011).

9. Cheung, L. W. et al. High frequency of PIK3R1 and PIK3R2 mutations in endometrial cancer elucidates a novel mechanism for regulation of PTEN protein stability. Cancer Discov. 1, 170-185 (2011).

10. Tate, J. G. et al. COSMIC: the Catalogue Of Somatic Mutations In Cancer. Nucleic Acids Res. 47, D941-d947 (2019).

11. Chang, M. T. et al. Identifying recurrent mutations in cancer reveals widespread lineage diversity and mutational specificity. Nat. Biotechnol. 34, 155-163 (2016).

12. Gao, J. et al. 3D clusters of somatic mutations in cancer reveal numerous rare mutations as functional targets. Genome Med. 9, 4 (2017).

13. Richards, S. et al. Standards and guidelines for the interpretation of sequence variants: a joint consensus recommendation of the American College of Medical Genetics and Genomics and the Association for Molecular Pathology. Genet. Med. 17, 405-423 (2015).

14. Engelman, J. A., Luo, J. \& Cantley, L. C. The evolution of phosphatidylinositol 3kinases as regulators of growth and metabolism. Nat. Rev. Genet. 7, 606-619 (2006).

15. Fruman, D. A. \& Bismuth, G. Fine tuning the immune response with PI3K. Immunol. Rev. 228, 253-272 (2009).

16. Rivière, J. B. et al. De novo germline and postzygotic mutations in AKT3, PIK3R2 and PIK3CA cause a spectrum of related megalencephaly syndromes. Nat. Genet. 44, 934-940 (2012).

17. Kurek, K. C. et al. Somatic mosaic activating mutations in PIK3CA cause CLOVES syndrome. Am. J. Hum. Genet. 90, 1108-1115 (2012).

18. Wentink, M. et al. Genetic defects in PI3K $\delta$ affect B-cell differentiation and maturation leading to hypogammaglobulineamia and recurrent infections. Clin. Immunol. 176, 77-86 (2017).

19. Sun, M., Hillmann, P., Hofmann, B. T., Hart, J. R. \& Vogt, P. K. Cancer-derived mutations in the regulatory subunit p85alpha of phosphoinositide 3-kinase function through the catalytic subunit p110alpha. Proc. Natl. Acad. Sci. USA 107, 15547-15552 (2010)

20. Oliver, M. D. et al. Insights into the pathological mechanisms of p85a mutations using a yeast-based phosphatidylinositol 3-kinase model. Biosci. Rep. 37, BSR20160258 (2017).

21. Fruman, D. A. \& Rommel, C. PI3K and cancer: lessons, challenges and opportunities. Nat. Rev. Drug Discov. 13, 140-156 (2014).

22. Coulter, T. I. et al. Clinical spectrum and features of activated phosphoinositide 3kinase $\delta$ syndrome: a large patient cohort study. J. Allergy Clin. Immunol. 139, 597-606.e594 (2017).

23. Lucas, C. L., Chandra, A., Nejentsev, S., Condliffe, A. M. \& Okkenhaug, K. PI3K $\delta$ and primary immunodeficiencies. Nat. Rev. Immunol. 16, 702-714 (2016).

24. Dornan, G. L., Siempelkamp, B. D., Jenkins, M. L., Vadas, O., Lucas, C. L. \& Burke, J. E. Conformational disruption of PI3K $\delta$ regulation by immunodeficiency mutations in PIK3CD and PIK3R1. Proc. Natl. Acad. Sci. USA 114, 1982-1987 (2017).

25. Jaiswal, B. S. et al. Somatic mutations in p85alpha promote tumorigenesis through class IA PI3K activation. Cancer Cell. 16, 463-474 (2009).

26. Dyment, D. A. et al. Mutations in PIK3R1 cause SHORT syndrome. Am. J. Hum. Genet. 93, 158-166 (2013).

27. Avila, M. et al. Clinical reappraisal of SHORT syndrome with PIK3R1 mutations: toward recommendation for molecular testing and management. Clin. Genet. 89, 501-506 (2016)

28. Bravo García-Morato, M. et al. Mutations in PIK3R1 can lead to APDS2, SHORT syndrome or a combination of the two. Clin. Immunol. 179, 77-80 (2017).

29. Cerami, E. et al. The cBio cancer genomics portal: an open platform for exploring multidimensional cancer genomics data. Cancer Discov. 2, 401-404 (2012).

30. Gao, J. et al. Integrative analysis of complex cancer genomics and clinical profiles using the cBioPortal. Sci. Signal. 6, pl1 (2013).

31. Ye, K. et al. Systematic discovery of complex insertions and deletions in human cancers. Nat. Med. 22, 97-104 (2016).

32. Ross, R. L., Burns, J. E., Taylor, C. F., Mellor, P., Anderson, D. H. \& Knowles, M. A. Identification of mutations in distinct regions of p85 alpha in urothelial cancer. PLoS One. 8, e84411 (2013).

33. Venot, Q. et al. Targeted therapy in patients with PIK3CA-related overgrowth syndrome. Nature. 558, 540-546 (2018).

\section{ACKNOWLEDGEMENTS}

The authors thank the participating individuals and their families described in this manuscript for their support and cooperation and the NIH Intramural Sequencing Center for performing NGS capture and sequencing. L.G.B. was supported by the intramural research program of the National Human Genome Research Institute, grants HG200328 and HG200388. R.K.S. was supported by the Wellcome Trust, grant 210752/Z/18/Z.

\section{AUTHOR CONTRIBUTIONS}

Conceptualization: C.E.C., B.A.D. Data curation: N.R.B., M.A., D.J.C. Formal analysis: C.E.C., M.Z., R.K.S., L.G.B., B.A.D. Methodology: C.E.C., J.H., V.M., L.G.B., B.A.D. Investigation: C.E.C., J.H., M.C., M.E., J.N.D., J.J.J., M.J.L., C.C.C., I.F., M.T., A.Z., H.C., L.L. T. Resources: D.J.C., J.H., M.C., M.E., J.J.J., M.J.L., O.M.T.D., C.C.C., I.F., M.T., A.Z., H.C., L.L.T. Validation: C.E.C., V.M., J.J.J., M.J.L., L.G.B. Visualization: C.E.C., K.V.C. Writing-original draft: C.E.C., MA, V.M., RKS, LGB, B.A.D. Writing-review and editing: C.E.C., R.K.S., L.G.B., B.A.D., O.M.T.D. Supervision: C.E.C., L.G.B., B.A.D.

\section{Compliance with ethical standards}

\section{ETHICS DECLARATION}

The work presented herein was considered by the institutional review boards (IRB) designated to oversee and monitor biomedical human subjects research at the $\mathrm{NIH}$, Washington University School of Medicine, and the University of Wisconsin. The limited, de-identified data associated with individuals contributed by Washington University School of Medicine were deemed as nonhuman subjects research by the IRB. Consent for participating individuals contributed by the $\mathrm{NIH}$ and through the multi-institutional network falling under the IRB of record through the University of Wisconsin, including publication and photography, was obtained.

\section{COMPETING INTERESTS}

L.G.B. has received in-kind research support from ArQule, Inc. (now wholly owned by Merck, Inc.), Novartis, and Pfizer Inc., royalties from Genentech, Inc, and honoraria from Cold Spring Harbor Press, as well as the following: Illumina Corp, Ethics Advisory Board. J.W.H. PierianDx, Knowledgebase Expert Panel member. B.A.D. has received in-kind research support from Venthera and research support for an investigator-initiated retrospective on oral propranolol from Pierre Fabre, and also reports the following: Venthera, Clinical Advisory Board; Consultant, Co-founder Pediatric Derm development, LLC. R.K.S. has received in-kind research support from Pfizer, Inc., and is a consultant for Novartis. L.L.T. has received research support from Ultragenyx Pharmaceutical. I.J.F. reports the following: Venthera, Consultant, Novartis, Consultant and Medical Advisory Board, Pfizer Inc., Data Safety Monitoring Boards. M.T. is a site principal investigator for Venthera. The other authors declare no competing interests.

\section{ADDITIONAL INFORMATION}

Supplementary information The online version contains supplementary material available at https://doi.org/10.1038/s41436-021-01211-z.

Correspondence and requests for materials should be addressed to B.A.D.

Reprints and permission information is available at http://www.nature.com/ reprints

Publisher's note Springer Nature remains neutral with regard to jurisdictional claims in published maps and institutional affiliations.

Open Access This article is licensed under a Creative Commons Attribution 4.0 International License, which permits use, sharing, adaptation, distribution and reproduction in any medium or format, as long as you give appropriate credit to the original author(s) and the source, provide a link to the Creative Commons license, and indicate if changes were made. The images or other third party material in this article are included in the article's Creative Commons license, unless indicated otherwise in a credit line to the material. If material is not included in the article's Creative Commons license and your intended use is not permitted by statutory regulation or exceeds the permitted use, you will need to obtain permission directly from the copyright holder. To view a copy of this license, visit http://creativecommons. org/licenses/by/4.0/.

(c) The Author(s) 2021 